Available online at_www.iponlinejournal.com

\title{
High grade pleomorphic sarcoma of penis: A rare clinical entity
}

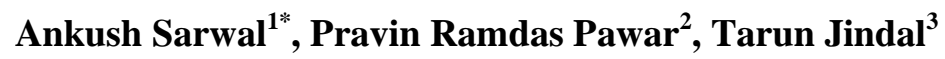 \\ ${ }^{1,2}$ Fellow, ${ }^{3}$ Consultant, Dept. of Uro-oncology, Tata Medical Centre, Kolkata, India
}

\begin{abstract}
The incidence of penile cancer has been reported to be around $0.005 \%$ in males, with less than $5 \%$ of then being sarcomas among all types of penile malignancies Primary penile sarcoma is a rare disease that affects men of all ages. Different subtypes of primary penile sarcoma exist, with the rarest being pleomorphic sarcoma. Only few cases have been reported on pleomorphic sarcoma of penis with undifferentiated type being most commonly reported. No report on high grade pleomorphic sarcoma has been previously reported in the English literature. We report an extremely rare case of undifferentiated pleomorphic sarcoma of the penis in a 62-year-old patient who initially presented with a recurrent or residual penile lesion. The tumor was surgically excised, but the patient experienced local recurrence and died of metastatic disease 18 months after initial presentation.
\end{abstract}

Keywords: Penile sarcoma penile cancer urogenital sarcoma.

\section{Introduction}

Primary penile sarcoma is one of the rare penile cancers. The incidence of penile cancer has been reported to be around $0.005 \%$ in males, with less than $5 \%$ of then being sarcomas among all types of penile malignancies.1 Vascular sarcomas are the most common type of penile sarcoma. Other rare types such as rhabdomyosarcomas, leiomyosarcomas, and fibrosarcomas have also been reported in literature. ${ }^{2}$ Very few reports on Pleomorphic Sarcoma of the Penis are there in literature and is regarded as rarest type of penile sarcoma. Due the rarity of the disease there are no guidelines and data on the treatment of penile sarcoma which complicates the decisionmaking process during initial therapy. Thus, the treatment for this condition remains controversial. ${ }^{3}$ Here we report the case of a 62-year-old male who treated for High grade pleomorphic sarcoma of penis.

\section{Case Report}

A 62-year-old man presented with a recurrent necrotising growth over the penis. He was operated for the same elsewhere 2 months ago. Initially he underwent Incisional biopsy at our centre. The histopathology examination (HPE) showed malignant spindle to epitheloid cells with a mitotic count of 1617 per high power field. Scattered lymphoid cells were also noted. Special stains such as PAS with diastase did bot highlight any intracytoplasmic inclusions. The tumour cells showed positivity for S100 but were negative for cytokeratin, CD 45, CD 34, Desmin CD117 and synaptophysin suggesting it to spindle cell sarcoma. Due to the recurrent nature and biopsy finding. Patient underwent Total penectomy with reconstruction with lateral thigh perforator flap and perineal urostomy. HPE of the specimen showed grey white tumour of size $9.6 \times 3.6 \times 3.3 \mathrm{~cm}$ with foci of haemorrhage and necrosis. Microscopic examination revealed malignant spindle cells with eosinophillic cytoplasm and hyperchromatic nuclei (Fig. 1). Lymphatic tumour emboli were seen with proximal, cavernosa, spongiosa and urethra margin free of tumour. Tumour cells were Vimentin positive and negative for S-100, CK, SMA, bcl-2 and CD-34 suggesting it to be High Grade Pleomorphic sarcoma of penis. Post operatively patient was discharged after 1 week and kept on follow-up. 6 months after the surgery patient reported to the hospital with complaints of bleeding from flap area which looked like a recurrence or organised hematoma. MRI of the

\footnotetext{
*Corresponding Author: Ankush Sarwal, Dept. of Uro-oncology, Tata Medical Centre, Kolkata, India Email: dravidavinu@gmail.com
} 
pelvis showed a recurrent mass at the root of penis and anterior wall of scrotum. Patient was informed about the recurrence of disease and medical oncology consultation was taken, who suggested no role of chemotherapy in current case scenario. Patient was kept on palliative management in form of analgesics and wound dressing. Eventually patient succumbed to the disease by 18 months of initial presentation.

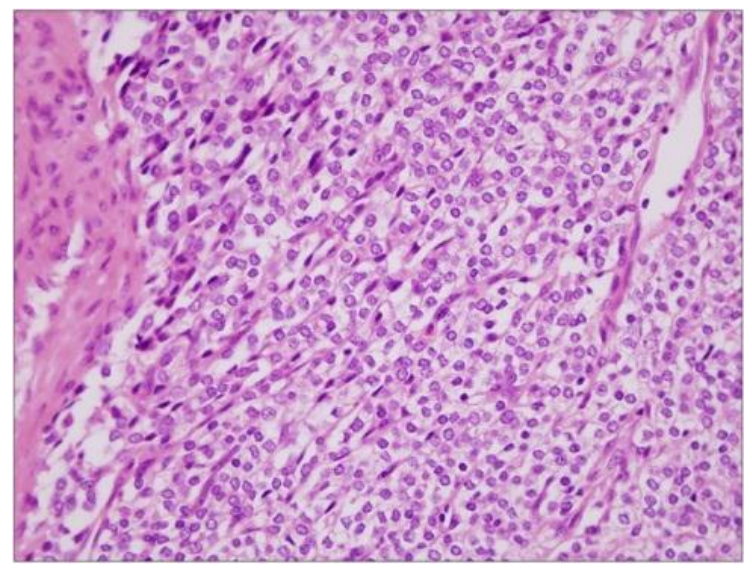

Fig. 1

\section{Discussion}

Penile cancer is uncommon and mostly consists squamous cell carcinoma reported incidence of 0.5 to 5 cases in every 100,000 males. ${ }^{1}$ Primary sarcoma of the penis is extremely uncommon and its etiology remains unknown. Soft tissue tumours of the penis consist mainly of vascular sarcomas followed by rhabdomyosarcomas, leiomyosarcomas, fibrosarcomas and Pleomorphic sarcoma being the rarest. Only few cases have been reported on pleomorphic sarcoma of penis with undifferentiated type being most commonly reported. No report on high grade pleomorphic sarcoma has been previously reported in the English literature. ${ }^{3,4}$ Common symptoms of penile sarcoma include penile nodularity or mass, urethral stenosis, penile curvature, and dysuria. The best approach for the management of these malignant penile lesions is early diagnosis and treatment before invasion occurs. ${ }^{5}$ Given the relative rarity of penile sarcoma no standardized treatment guideline or protocol exists for them. Thus, the therapeutic approach to such cases is very challenging and remains controversial. Most of the case reports on penile sarcoma have used total penectomy with or without associated radiation and chemotherapy as the treatment modality. ${ }^{6}$ High local recurrence rate after surgery of up to $80 \%$ and occurrence rates of pulmonary and regional lymph node metastasis of up to $51 \%$ and $30 \%$, respectively are seen in patients with penile sarcoma. ${ }^{4,7}$ Regardless of the therapeutic modality used, the majority of patients die in less than two years due to systemic dissemination. ${ }^{6}$ The most appropriate approach to treating such conditions remains to be determined. Further studies are needed to determine the standard therapeutic modalities for these diseases.

\section{Conclusion}

Primary penile sarcoma is a rare disease, and limited data are available to guide treatment. Resection with or without adjuvant therapies is the standard treatment for the localized disease, whereas patients with advanced disease may be treated with radiation or systemic therapies. However, surgery is considered the only curative treatment, and, therefore, early recognition and surgical excision may improve the outcomes of patients with this disease. Further studies are needed to determine the standard therapeutic modalities for these diseases.

\section{Source of Funding}

None.

\section{Conflict of Interest}

None.

\section{References}

1. Katsikas VS, Kalyvas KD, Ioannidis SS, Papathanasiou MV, Panagiotopoulou KP, Hitiroglou PM, et al. Leiomyosarcoma of the penis. Sarcoma 2002;6:75-7.

2. Dominici A, Delle Rose A, Stomaci N, Pugliese L, Posti A, Nesi G. A rare case of leiomyosarcoma of the penis with a reappraisal of the literature. Int J Urol 2004;11:440-4.

3. Yoo HS, Satti S, Matrana M. Primary Undifferentiated Pleomorphic Sarcoma of the Penis. Ochsner J 2017;17(4):434-7.

4. Choi YH, Kim HW, Ahn JH, et al. Primary undifferentiated penile sarcoma in adolescence. Korean J Urol 2012;53(10):733-6. doi: 10.4111/kju.2012.53.10.733.

5. Shabbir M, Kayes O, Minhas S. Challenges and controversies in the management of penile cancer. Nat Rev Urol 2014;11(12):702-11. doi: 10.1038/nrurol.2014.307 
6. Antunes AA, Nesrallah LJ, Gonçalves PD, Ferreira YA, Campagnari JC Srougi M. Deep-seated sarcomas of the penis. Int Braz J Urol 2005;31:245-50.

7. Katona TM, Lopez-Beltran A, MacLennan GT, Cheng L, Montironi R, Cheng L et al. Soft tissue tumors of the penis: a review. Anal Quant Cytol Histol 2006;28(4):193-206.

How to cite this article: Sarwal A, Pawar PR, Jindal T. High grade pleomorphic sarcoma of penis: A rare clinical entity. J Nutr, Metab Health Sci, 2019;2(3):8991. 